\title{
Radiation pneumonitis complicating mediastinal radiotherapy postpneumonectomy
}

\author{
T. Shulimzon*, S. Apter**, R. Weitzen+, A. Yellin++, H.J. Brenner+, A. Wollner*
}

Radiation pneumonitis complicating mediastinal radiotherapy postpneumonectomy. $T$. Shulimzon, S. Apter, R. Weitzen, A. Yellin, H.J. Brenner, A. Wollner. CERS Journals Ltd 1996.

ABSTRACT: Radiation pneumonitis is a well-characterized clinicopathological syndrome. The severity of radiation-induced lung injury correlates, among other factors, with the extent of lung volume incorporated within the field of radiation.

The present article describes the cases of two patients with radiation pneumonitis following pneumonectomy and mediastinal radiotherapy. Postpneumonectomy pulmonary-mediastinal shift of the remaining lung towards the operated side, with inclusion of lung parenchyma within the "mediastinal" radiation portals, resulted in a substantial (albeit clinically unsuspected) radiation pneumonitis.

Chest computed tomography in the postpneumonectomy patient may be helpful to evaluate the degree of pulmonary-mediastinal shift and optimization of the radiotherapy field.

Eur Respir J., 1996, 9, 2697-2699.
*Division of Respiratory Medicine, and Depts of $* *$ Radiology, ${ }^{+}$Oncology and ${ }^{++}$Thoracic Surgery, The Chaim Sheba Medical Center, affiliated to the Tel-Aviv University Sackler Medical School, TelHashomer, Israel.

Correspondence: A. Wollner, Division of Respiratory Medicine, Sheba Medical Center, Tel-Hashomer 52621, Israel.

Keywords: Chest computed tomography, lung cancer, mediastinal radiotherapy, postpneumonectomy pulmonary-mediastinal shift, radiation pneumonitis

Received: April 251996

Accepted after revision August 81996
Radiation therapy following lung surgery may improve survival in some subsets of patients with lung cancer [1]. The principles of this treatment are to control the local spread of the tumour, with minimal injury to the surrounding lung parenchyma. These principles are especially valid in the postpneumonectomy patient. In the postpneumonectomy state, the mediastinum and remaining lung are deviated towards the "vacant" hemithorax. This pulmonary-mediastinal shift should be taken into account when planning mediastinal radiation, in order to minimize potential morbidity.

\section{Case report}

\section{Case 1}

A 51 year old male underwent a left pneumonectomy for primary squamous cell carcinoma. Postoperatively, he was staged as $\mathrm{T} 2 \mathrm{~N} 2 \mathrm{M} 0$ (tumour, node, metastasis classification), and received anterior and posterior field mediastinal radiation for 25 days to a total of 4,000 cGy. The right lung appeared normal on a chest radiographic image that was performed before initiation of radiotherapy.

Three weeks after completion of radiotherapy, the patient developed cough, dyspnoea and fever. On physical examination, he appeared ill, but there were no other abnormal findings. Chest radiography revealed displacement of the right lung and mediastinum to the left, with opacification of the right upper paramediastinal area and perivascular haziness in the right lower paramediastinal region (fig. 1a). A work-up for underlying infection, including blood and sputum tests as well as a percutaneous aspirate from the left pleural cavity, was negative. Pulmonary function tests revealed a total lung capacity (TLC) of $48 \%$ predicted, residual volume (RV) of $59 \%$ predicted, and transfer factor of the lung for carbon monoxide ( $T \mathrm{~L}, \mathrm{CO}) / \mathrm{TLC}$ ratio of $35 \%$. Galium scan showed upake over the right lung, mainly in the retrosternal area. Chest computed tomography (CT) demonstrated pulmonary-mediastinal shift to the left, with a substantial infiltrate involving the retrosternal and right parasternal lung fields, with a "straight edge effect" (fig. $1 \mathrm{~b}$ and $\mathrm{c})$.

A clinical diagnosis of radiation pneumonitis affecting the retrosternally shifted lung was made, and prednisone, at a dose of $60 \mathrm{mg} \cdot \mathrm{day}^{-1}$, was initiated. A marked clinical and radiological improvement was noted within 3 weeks, and the repeated $T \mathrm{~L}, \mathrm{CO} / \mathrm{TLC}$ increased to $68 \%$.

\section{Case 2}

A 50 year old female underwent a left mastectomy for adenocarcinoma in 1985, and received postoperative radiotherapy using a single supraclavicular and two opposing tangential fields across the left chest wall to a total of 4,000 cGy. A left pneumonectomy for an adenocarcinoma of the lung was performed in 1992. Postoperatively, the patient was staged as T2N2M0, and received anterior and posterior field mediastinal radiation with 3,000 cGy in 10 doses. Chest radiographic image obtained before radiotherapy showed a normal right lung.

Four weeks after termination of radiotherapy, the patient developed dyspnoea, cough, fever and chest pain. 
a)

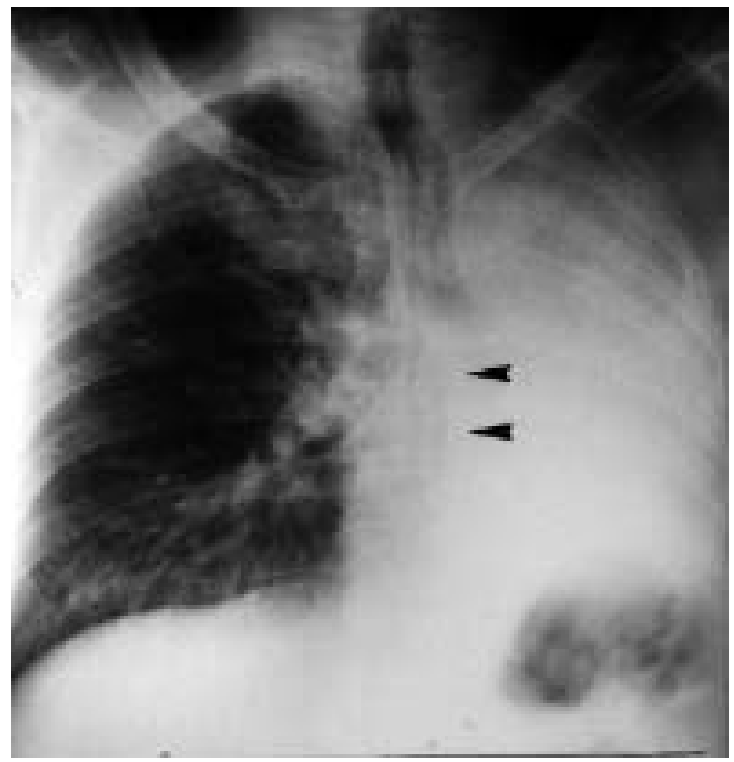

b)

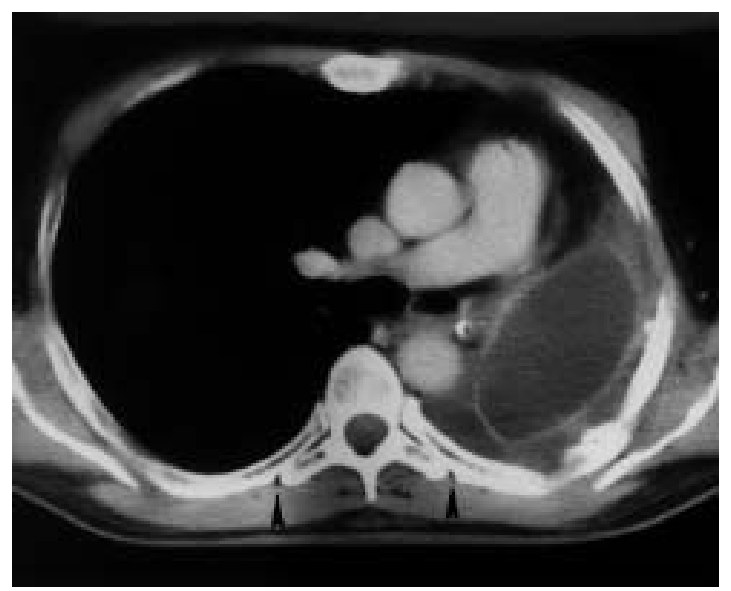

c)

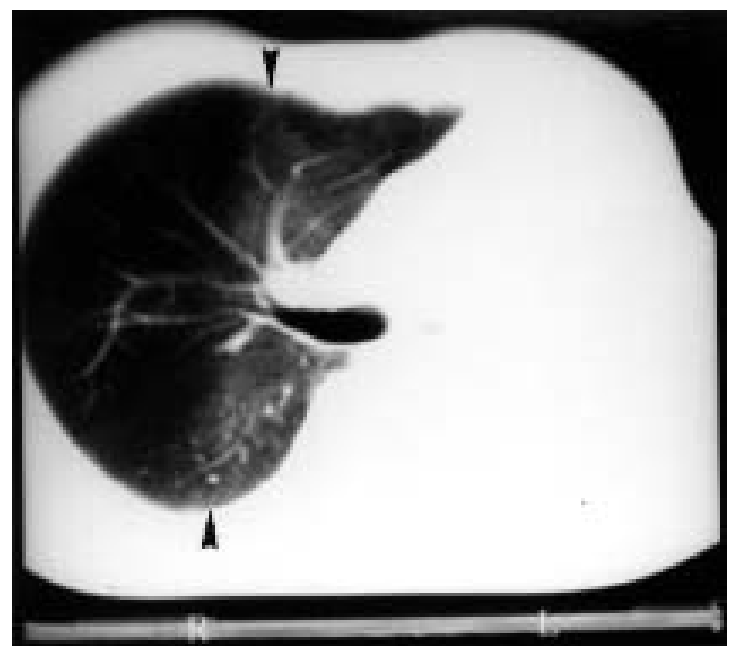

Fig. 1. - a) Chest radiograph obtained 3 weeks after completion of radiotherapy, showing an opacity in the right upper paramediastinal region and haziness around the vessels in the lower paramediastinal region. Deviation of the right lung towards the unoccupied hemithorax is marked with arrowheads. b) Computed tomography (CT) scan, showing the pulmonary-mediastinal shift. The mediastinal radiation field is confined between the transverse processes of the vertebral bodies (marked by arrowheads). c) CT scan demonstrating the infiltrate, with a "straight edge effect" (arrowheads) consistent with radiation pneumonitis.
Physical examination revealed a to-and-fro pericardial friction rub, with no other abnormal findings. Echocardiography revealed pericardial effusion. Pulmonary-mediastinal displacement to the left, with minimal haziness around the vessels of the right upper and lower paramediastinal areas, were seen on chest radiographic image (fig. 2a). Chest CT revealed opacification of the retrosternally-shifted lung field (fig. $2 b$ and c). Pulmonary

a)

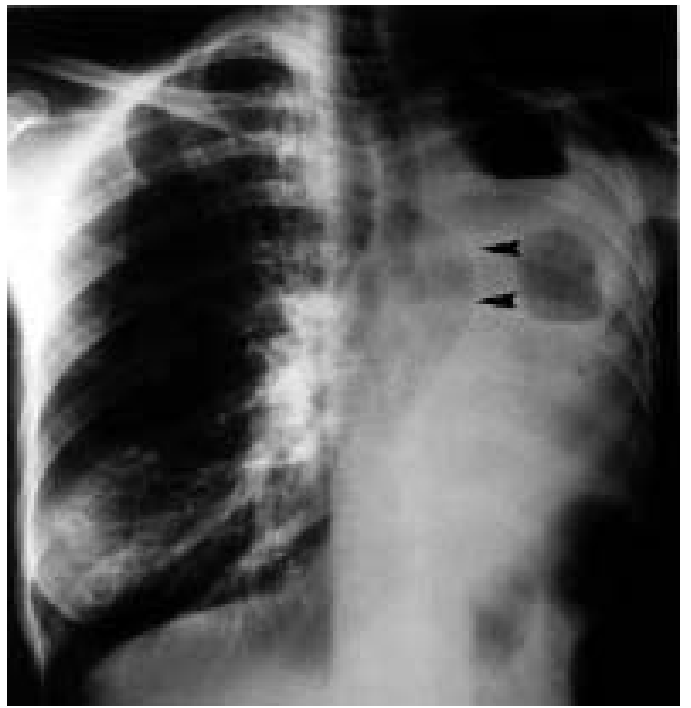

b)

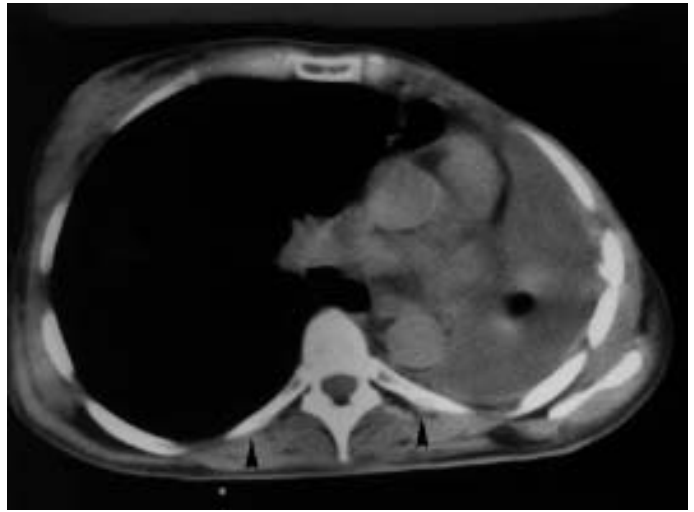

c)

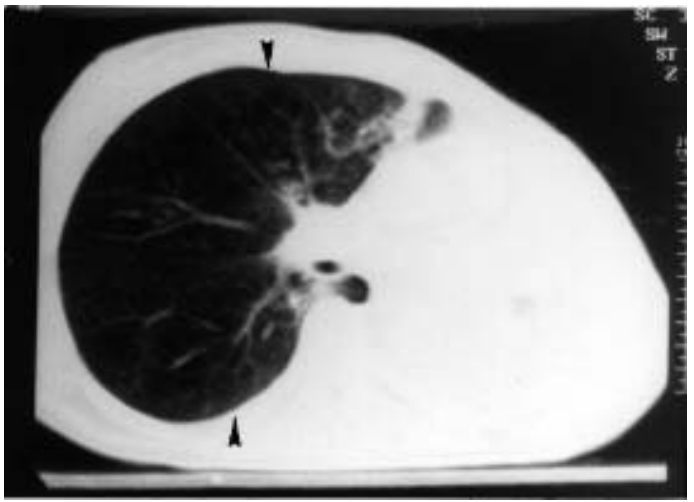

Fig. 2. - a) Chest radiograph obtained 4 weeks after completion of radiotherapy. Note deviation of the right lung towards the unoccupied hemithorax (marked with arrowheads). b) Computed tomography $(\mathrm{CT})$ scan showing the pulmonary-mediastinal shift. The mediastinal radiation field is confined between the transverse processes of the vertebral bodies (marked by arrowheads). c) CT scan displaying haziness confined to the mediastinal radiotherapy field (arrowheads). 
function tests revealed a $T \mathrm{~L}, \mathrm{CO} / \mathrm{TLC}$ of $37 \%$. Blood and sputum bacteriological tests were negative.

Oral prednisone, at a dose of $60 \mathrm{mg} \cdot \mathrm{day}^{-1}$, was initiated with resolution of the pericarditis. Pulmonary symptoms and chest radiographic image improved gradually over the next 3 weeks, but without any change in diffusion capacity.

\section{Discussion}

The two cases reported received postpneumonectomy mediastinal radiotherapy for mediastinal lymph node involvement.

Clinical radiation pneumonitis complicating mediastinal radiotherapy is infrequent. In the series of 592 patients with Hodgkin's disease and lymphoma, reported by KAPLAN and STEWART [2] in 1973, mediastinal radiotherapy resulted in clinical radiation pneumonitis in only $6.4 \%$.

Simulation for mediastinal portals is usually performed under fluoroscopy, in order to confine the radiation field to the transverse processes of the vertebral bodies. Deviation of the mediastinum and lung towards the operated side is well-known following pneumonectomy. This retrosternal shift may have a dual effect, namely, a substantial portion of lung volume is inadvertently included within the "mediastinal" radiation port, and, in addition, the sternal and vertebral silhouettes may significantly obscure the radiographic manifestations of radiation pneumonitis (figs. 1 and 2). Lack of clinical awareness of this complication might result in an extensive work-up for pulmonary infection as well as recurrent malignancy. Chest CT proved helpful to evaluate this retrosternal pulmonary-mediastinal shift and associated radiation pneumonitis.

We would like to suggest that the current practice of simulation for mediastinal portals without appreciation of postpneumonectomy pulmonary-mediastinal shift may result in suboptimal irradiation of the cancerous lymph nodes in their shifted location, whilst at the same time exposing the healthy retrosternally-deviated single lung to unnecessary radiation. A different practice of simulation based on CT treatment planning can be more helpful to optimize the radiation plan. Radiotherapy, using anterior and posterior fields during half of the treatment in addition to three oblique fields entering through the thorax where the lung is absent, may result in low doses of radiation to the remaining lung [3].

To the best of our knowledge, the present observation has not been reported previously [4-7]. It is suggested that increased awareness of this postpneumonectomy pulmonary-mediastinal shift and computed tomography based radiation planning may maximize the dose to the shifted mediastinum, whilst minimizing radiation to the remaining lung.

Prospective studies to investigate the importance of postpneumonectomy lung shift and its correlation to the risk and severity of radiation pneumonitis are needed.

Acknowledgements: The authors wish to thank R.J. Shiner and I. Ben-Dov for their critical review of the manuscript.

\section{References}

1. Murren JR, Buzaid AC. Chemotherapy and radiation for the treatment of non-small cell lung cancer. Clin Chest Med 1993; 14: 161-172.

2. Kaplan HS, Stewart JR. Complications of intensive megavoltage radiotherapy for Hodgkin's disease. Natl Cancer Inst Monogr 1973; 36: 439-444.

3. Bentel GC, Nelson CE, Noell KT (eds). In: Treatment planning and dose calculation in radiation oncology. Oxford, Pergamon Press, 1989.

4. Gross NJ. Pulmonary effects of radiation therapy. Ann Intern Med 1977; 86: 81-92.

5. Rosiello RA, Merrill WW. Radiation-induced lung injury. Clin Chest Med 1990; 11: 65-71.

6. Davis SD, Yankelevitz DF, Henschke CI. Radiation effects on the lung: clinical features, pathology, and imaging findings. AJR 1992; 159: 1157-1164.

7. Marks LB. The pulmonary effects of thoracic irradiation. Oncology (Huntingt) 1994; 8: 89-106. 